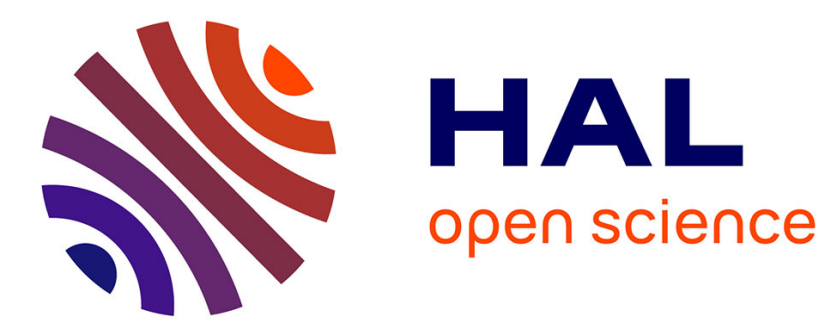

\title{
Prise en compte du procédé de mise en forme d'un brancard dans les paramètres matériaux de simulation au CRASH
}

\author{
E. Blouet, J. Lagrange, Loick Verger, G. Le Borgne
}

\section{To cite this version:}

E. Blouet, J. Lagrange, Loick Verger, G. Le Borgne. Prise en compte du procédé de mise en forme d'un brancard dans les paramètres matériaux de simulation au CRASH. Journal de Physique IV Proceedings, 1997, 07 (C3), pp.C3-945-C3-950. 10.1051/jp4:19973159 . jpa-00255448

\section{HAL Id: jpa-00255448 https://hal.science/jpa-00255448}

Submitted on 1 Jan 1997

HAL is a multi-disciplinary open access archive for the deposit and dissemination of scientific research documents, whether they are published or not. The documents may come from teaching and research institutions in France or abroad, or from public or private research centers.
L'archive ouverte pluridisciplinaire HAL, est destinée au dépôt et à la diffusion de documents scientifiques de niveau recherche, publiés ou non, émanant des établissements d'enseignement et de recherche français ou étrangers, des laboratoires publics ou privés. 


\title{
Prise en compte du procédé de mise en forme d'un brancard dans les paramètres matériaux de simulation au CRASH
}

\author{
E. Blouet, J.L. Lagrange*, L. Verger et G. Le Borgne \\ DRAS centre SAMM, Chemin de la Malmaison, 91570 Bièvres, France \\ *DETA/MXT, Route de Gisy, 78140 Vélizy-Villacoublay, France
}

\begin{abstract}
Résumé. La mise en forme industrielle d'un brancard est obtenue par emboutissage. Le matériau subit alors sur certaines faces de la pièce une prédéformation dont on veut évaluer l'influence : sur le comportement mécanique du matériau, sur les performances du brancard au CRASH. On a pour cela comparé le comportement de brancards emboutis avec celui de brancards mis en forme par pliage. Une caractérisation statique et dynamique du matériau in situ a permis d'identifier les paramètres de la loi élastoplastique du logiciel RADIOSS. Les calculs comparatifs utilisant ces différentes lois sont validés par des essais d'écrasement dynamique.
\end{abstract}

\begin{abstract}
The shape of a longitudinal steel beam is industrially obtained by stamping. The material is then submitted to a sever predeformation on certain faces of the beam. In order to estimate its influence on the mechanical behaviour of the material and on the crushing performance of the beams, we compare the behaviour of stamped beams to that of folded ones. Static and dynamic tests have been conducted to fit the elastoplastic law used by the crushing simulation code RADIOSS. Calculations have been carried out with the resulting laws and dynamic crushing tests have been performed as a validation of these results.
\end{abstract}

\section{INTRODUCTION}

Le procédé de mise en forme par emboutissage induit un état du matériau complexe, hétérogène et anisotrope. L'épaisseur des faces embouties diminue, les propriétés mécaniques du matériau augmentent. L'étude présentée dans cet article tend à évaluer sur une sous-structure particulière de la carrosserie automobile l'importance de ces modifications et leur influence sur le comportement de la pièce au crash. On s'attachera en particulier à déterminer s'il est opportun de prendre en compte ces phénomènes lors du dimensionnement de structure en Bureau d'Etudes.

La sous-structure constituant notre référence est un prototype de longeron avant (pièce appelée brancard) en acier extra-doux. On constitue un lot mis en forme par emboutissage et un lot mis en forme par pliage. La démarche adoptée ensuite peut être schématisée comme suit :

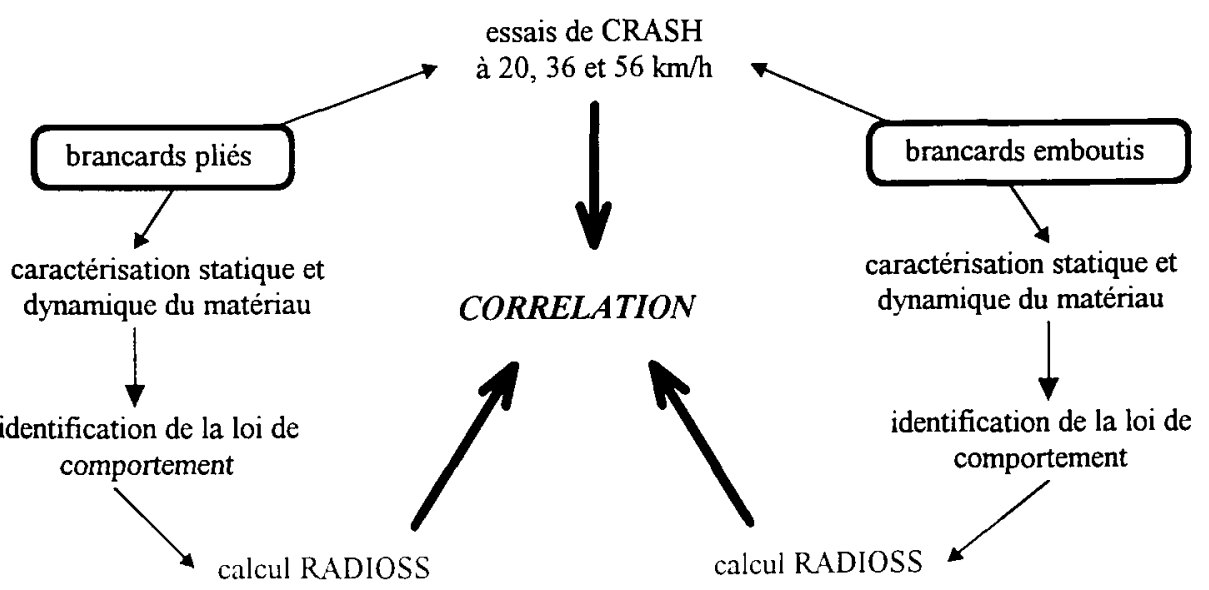




\section{CARACTERISATION EXPERIMENTALE DU MATERIAU}

\subsection{Nature des essais}

Des éprouvettes sont prélevées : . sur la tôle brute de laminage ;

. sur les différentes faces des brancards pliés et emboutis.

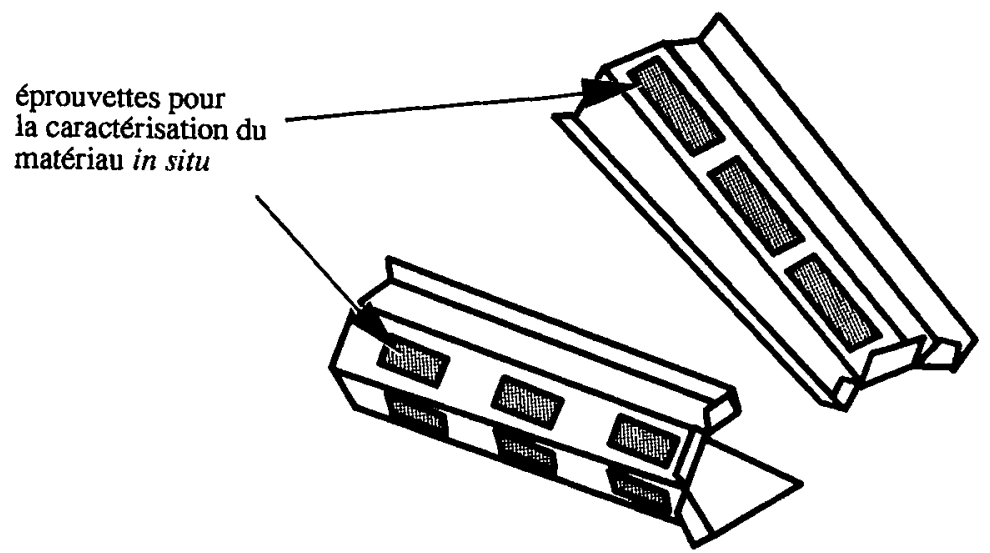

Les essais de traction quasi-statique sont menés sur une machine de traction traditionnelle. Les essais à vitesses moyennes (entre $0.25 / \mathrm{s}$ et $20 / \mathrm{s}$ ) proviennent d'une machine de traction rapide du laboratoire de mécanique de l'Ecole Centrale de Paris. Enfin les essais de compression dynamique pour les vitesses supérieures à 50 /s sont effectués sur un dispositif de barres d'Hopkinson au Laboratoire de Mécanique des Solides de l'Ecole Polytechnique.

\subsection{Influence de l'emboutissage en statique}

Pour une vitesse moyenne de $10^{-3} / \mathrm{s}$, les résultats des premières tractions donnent :

Tableau 1 : Quelques valeurs de caractérisation in situ de l'acier constituant les brancards

\begin{tabular}{|c|c|}
\hline & Reteve expertmetitat \\
\hline tôle initiale et brancard plié & ic Re 0.2\% \\
\hline fond du brancard embouti & $170 \mathrm{MPa}$ \\
\hline côté peu embouti du brancard & $194 \mathrm{MPa}$ \\
\hline $\begin{array}{c}\text { côté fortement embouti du } \\
\text { brancard }\end{array}$ & $209 \mathrm{MPa}$ \\
\hline
\end{tabular}

On remarque tout d'abord que le matériau des brancards pliés ainsi que des fonds de coquille des brancards emboutis ont un comportement très proche de la tôle initiale.

Malgré une diminution d'épaisseur assez modeste (pas plus de $8 \%$ de perte d'épaisseur), le comportement des zones fortement embouties du brancard est radicalement différent et la limite d'élasticité augmente de près de $80 \%$. 


\subsection{Prise en compte de l'influence de la vitesse}

L'acier extra-doux qui constitue les brancards testés est très sensible à la vitesse de déformation. La limite d'élasticité augmente énormément alors que les paramètres d'écrouissage diminuent. Les essais de compression dynamique mettent en évidence cette dépendance linéaire au logarithme de la vitesse au-delà de $50 / \mathrm{s}$. La figure 1 permet de constater une dépendance à peu près analogue pour la tôle initiale (ou tôle du brancard plié) et la tôle des côtés emboutis.

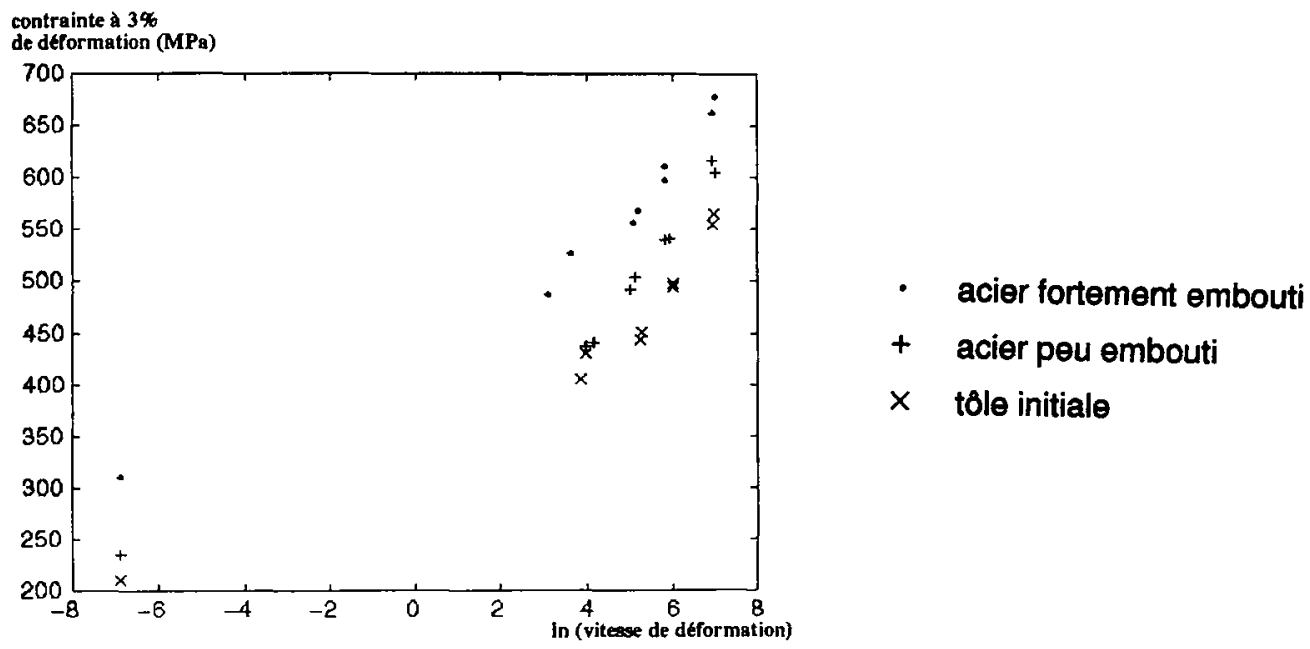

Figure 1 : Influence de la vitesse de déformation sur la contrainte à $3 \%$ de déformation plastique

\subsection{Identification de la loi de comportement}

La loi élastoplastique disponible dans le logiciel de simulation dynamique RADIOSS utilisé à PSA permet la prise en compte de l'influence de la vitesse grâce au coefficient multiplicatif de Johnson-Cook appliqué au modèle de Ludwik :

$$
\begin{cases}\sigma=\left(A+B \cdot \varepsilon_{p}^{n}\right) & \text { si } \dot{\varepsilon} \leq \dot{\varepsilon}_{0} \\ \sigma=\left(A+B \cdot \varepsilon_{p}^{n}\right) \cdot\left(1+C \cdot \ln \left[\frac{\dot{\varepsilon}}{\dot{\varepsilon}_{0}}\right]\right) & \text { si } \dot{\varepsilon}>\dot{\varepsilon}_{0}\end{cases}
$$

où $\sigma$ est la contrainte, $\varepsilon_{p}$ la déformation plastique, $\dot{\varepsilon}$ la vitesse de déformation.

$C$ et $\dot{\varepsilon}_{0}$ sont les deux paramètres qui définissent la dépendance bilinéaire au logarithme de la vitesse de déformation.

Les caractérisations statiques et dynamiques effectuées sur des échantillons de brancards pliés et emboutis aboutissent à l'identification de trois jeux complets de paramètres :

- une loi pour la tôle initiale, applicable à toutes les faces des brancards pliés et aux fond de coquille des brancards emboutis ;

- une loi pour les zones légèrement prédéformées des brancards emboutis ;

- une loi pour les zones très sollicitées lors de l'emboutissage. 


\section{COMPARAISON DU COMPORTEMENT AU CRASH DES BRANCARDS}

\subsection{Résultats expérimentaux}

Les essais d'écrasement de brancards sont menés dans les laboratoires du sidérurgiste COCKERILLSAMBRE (Liège). La pièce est fixée en position horizontale sur une structure rigide, un chariot mu par une catapulte vient percuter le brancard à la vitesse préalablement choisie.

Les modalités adoptées pour cette campagne d'essais sont les suivantes :

\begin{tabular}{|c|c|c|c|c|c|c|}
\hline Wirse erf forme & Nombre & d'impact (lam/) & $\begin{array}{l}\text { Masse dit: } \\
\text { chariot(kg) }\end{array}$ & $\begin{array}{l}\text { Bfiergie, } \\
\text { foumie (1) }\end{array}$ & mex 6 (a) & $\begin{array}{l}\text { Effort moyen } \\
(\mathrm{kN})\end{array}$ \\
\hline \multirow{3}{*}{ pliage } & 3 & 20 & 1020 & 16165 & 187 & 84.7 \\
\hline & 3 & 36 & 396 & 19986 & 195 & 85.2 \\
\hline & 3 & 57 & 128 & 15750 & 258 & 94.1 \\
\hline \multirow{3}{*}{ emboutissage } & 3 & 20 & 1020 & 15579 & 201 & 87.6 \\
\hline & 3 & 36 & 396 & 19859 & 232 & 94.1 \\
\hline & 3 & 57 & 128 & 15777 & 282 & 100 \\
\hline
\end{tabular}

L'effort moyen présenté ici correspond à l'effort relevé sur les 150 premiers mm d'écrasement. Ce choix a été fixé afin d'étudier un paramètre indépendant de l'énergie d'impact et des problèmes de stabilité de la pièce testée qui apparaissent en fin de choc.

L'effort max. est celui du pic d'entrée. Il est donné à titre indicatif. Ce n'est pas un paramètre de corrélation important car sa mesure est perturbée par des oscillations sur les courbes d'essai

Ces résultats amènent deux remarques essentielles

- l'emboutissage favorise un écrasement plus stable (moins de flambage) ainsi qu'un effort moyen supérieur (gain en effort moyen de $3.4 \%$ à $20 \mathrm{~km} / \mathrm{h}, 10.4 \%$ à $36 \mathrm{~km} / \mathrm{h}, 6.3 \%$ à $57 \mathrm{~km} / \mathrm{h}$ )

- le pic d'entrée des essais sur brancards emboutis est également plus élevé que sur les brancards pliés (gain allant jusqu'à 19\%).

Il existe donc une augmentation notable des performances au crash des brancards avec l'emboutissage. Celle-ci reste cependant faible devant l'augmentation des propriétés mécaniques du matériau observé dans le chapitre précédent.

\subsection{Simulation}

\subsubsection{Le modèle de brancard}

Le modèle reprend la géométrie des pièces d'essais. Il est maillé par des éléments coques d'épaisseur uniforme. Pour les modèles de brancards pliés, la loi appliquée sur toute la pièce est la loi de la tôle initiale. Pour les modèles emboutis, à chaque face du brancard est attribuée la loi matériau correspondant à son état moyen de prédéformation :

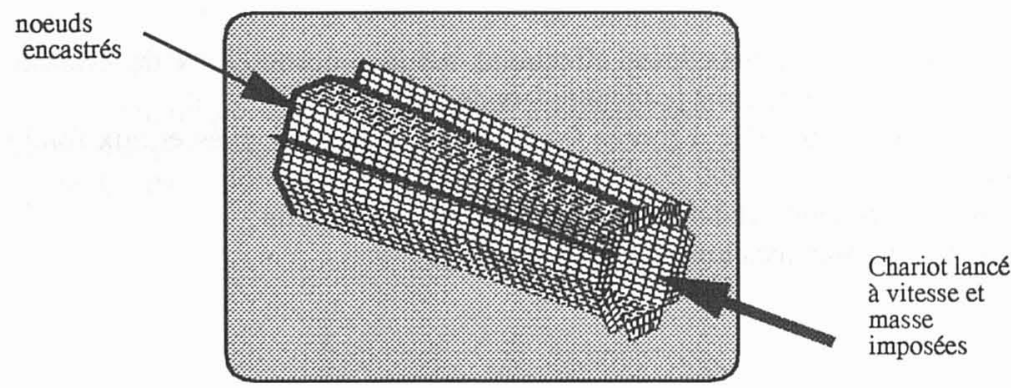




\subsubsection{Comparaison essais/calculs}

Chaque modalité d'essai est simulé sur RADIOSS. Les déformées obtenues, les niveaux d'effort relevés, permettent une comparaison essai/calcul pour chaque configuration :

\begin{tabular}{|l|c|c|c|}
\hline \multirow{2}{*}{ mise en forne } & $\begin{array}{c}\text { yitesse d impact } \\
\text { (knu) }\end{array}$ & $\begin{array}{c}\text { effort moyen } \\
\text { (kN) experience }\end{array}$ & $\begin{array}{c}\text { comparaison } \\
\text { essal / calcul }\end{array}$ \\
\hline \multirow{3}{*}{ pliage } & 20 & 84.7 & $3.2 \%$ \\
\cline { 2 - 4 } & 36 & 85.2 & $14 \%$ \\
\hline \multirow{3}{*}{ emboutissage } & 57 & 94.1 & $10.3 \%$ \\
\cline { 2 - 4 } & 20 & 87.6 & $4.5 \%$ \\
\cline { 2 - 4 } & 36 & 94.1 & $11.7 \%$ \\
\hline
\end{tabular}

L'effort moyen semble légèrement surestimé au calcul (décalage dû aux compromis imposés par la forme bilinéaire de la loi de comportement identifiée). Toutefois, le gain en effort moyen entre modèle plié et modèle embouti trouvé au calcul est très satisfaisant (respectivement de $4.7 \%$ à $20 \mathrm{~km} / \mathrm{h}, 8.2 \%$ à 36 $\mathrm{km} / \mathrm{h}, 1,0 \%$ à $57 \mathrm{~km} / \mathrm{h}$ ). On est donc parvenu, en partant de la caractérisation du matériau sur quelques zones prélevées sur les brancards, à reproduire au calcul l'influence de la mise en forme sur le comportement au choc de la structure.

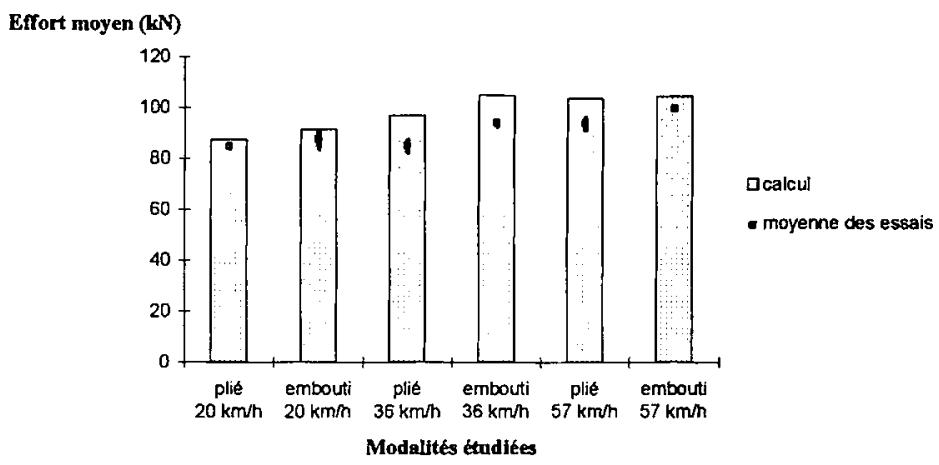

Figure 2 : Comparaison des résultats essais/calculs en effort moyen pour chaque modalité

Cependant, le gain lié à la mise en forme reste comparable aux écarts généralement admis en calcul de crash. On peut noter que le calcul réalisé avec la loi identifiée sur le matériau de base permet de bien rendre compte du comportement d'un brancard embouti (c.f. figure 3 page suivante).

\section{CONCLUSION}

L'étude ci-dessus présente une démarche de caractérisation du matériau in situ sur des pièces après mise en forme. Nous avons mis en évidence la capacité du modèle RADIOSS à restituer la modification du comportement de la structure liée à un état hétérogène du matériau. 
Dans l'optique de la simulation du comportement global d'une structure pour le bureau d'étude, ces résultats fournissent des informations supplémentaires. Il est à noter que la prise en compte de l'état du matériau in situ n'a pas amélioré la corrélation entre modèle et pièce emboutie. La simulation de l'écrasement dynamique d'une pièce emboutie avec une loi de comportement unique identifiée à partir de la tôle initiale foumit dans notre cas des résultats largement satisfaisants à l'échelle de la structure.

\section{REMERCIEMENTS}

Nous remercions les laboratoires de COCKERILL-SAMBRE pour les données expérimentales qu'ils ont fournies à notre étude.
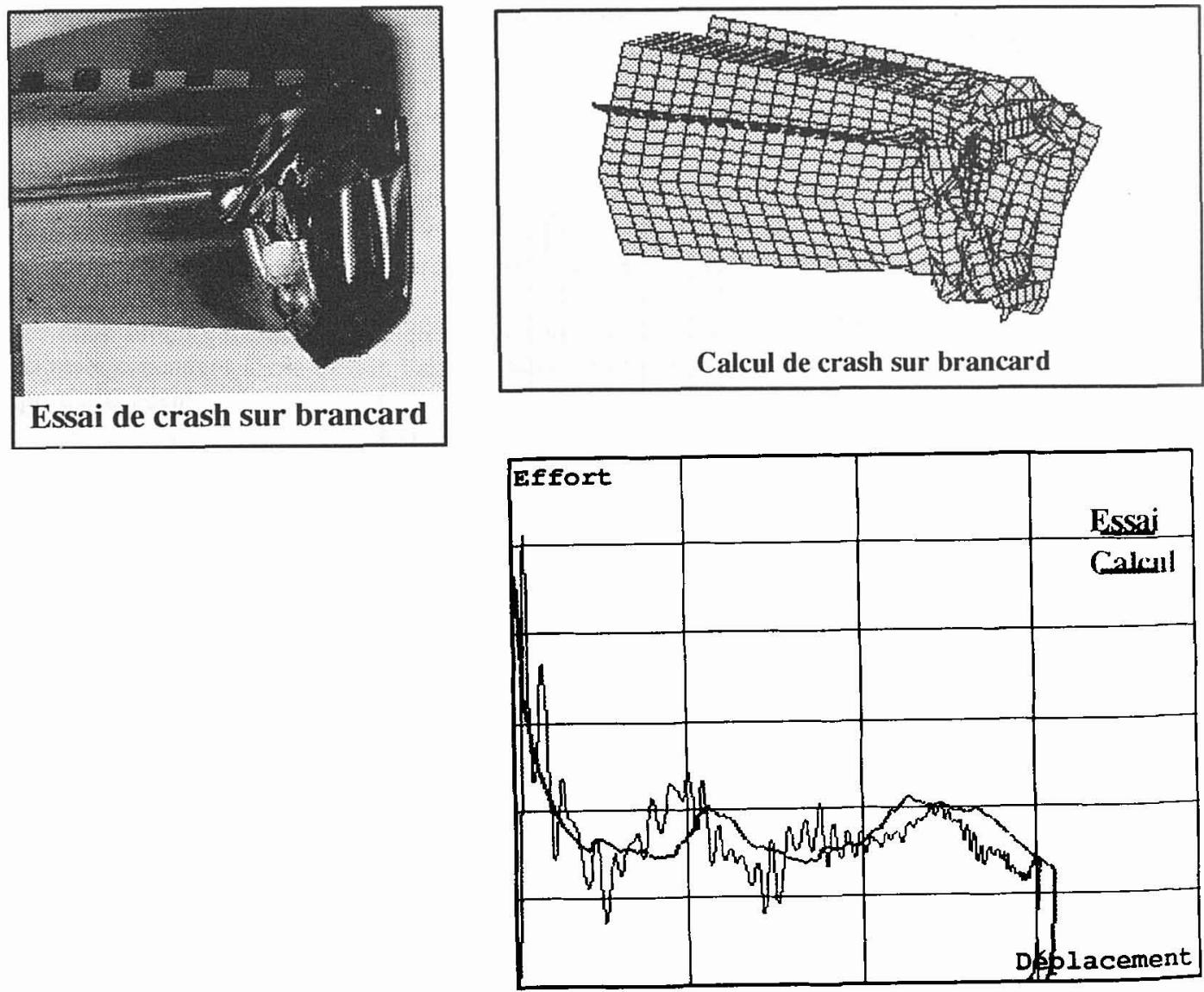

Figure 3 : Comparaison essai/calcul pour un brancard embouti modélisé avec la loi de la tôle initiale 